"This is the peer reviewed version of the following article: Ao, Z. and Li, S. (2014), Electric field manipulated reversible hydrogen storage in graphene studied by DFT calculations. Phys. Status Solidi A, 211: 351-356, which has been published in final form at 10.1002/pssa.201330129. This article may be used for non-commercial purposes in accordance with Wiley Terms and Conditions for Self-Archiving." 


\title{
Electric field manipulated reversible hydrogen storage in graphene studied by DFT calculations
}

\author{
Zhimin $\mathrm{Ao}^{*}, 1,2$ and Sean $\mathrm{Li}^{1}$ \\ ${ }^{1}$ School of Materials Science and Engineering, The University of New South Wales, Sydney, NSW 2052, Australia \\ ${ }^{2}$ Centre for Clean Energy Technology, School of Chemistry and Forensic Science, University of Technology, Sydney, PO Box 123 , \\ Broadway, Sydney, NSW 2007, Australia
}

Received ZZZ, revised ZZZ, accepted ZZZ

Published online ZZZ (Dates will be provided by the publisher.)

Keywords Graphene, Hydrogen Storage, Electric Field, Density Functional Theory.

* Corresponding author: e-mail Zhimi.Ao@uts.edu.au, Phone: +61-2-95141722, Fax: +61-2-95141460

\begin{abstract}
Enhancement of hydrogen storage capacity is a great challenge that the research community is facing. The challenge lies on the fact of interdependence of hydrogen storage and release processes. It presents that the hydrogen release would be difficult if the hydrogen can be stored easily or vice versa. This work strategically tackles this critical issue through density functional theory calculations by applying defect engineering to graphene, as well as by changing the hydrogenation/dehydrogenation and hydrogen diffusion chemical potentials via applying electric field. It is found that hydrogen molecules are dis-
\end{abstract}

\section{Introduction}

In recent years, hydrogen-based fuel systems have been considered to be a highly important topic of research for future energy schemes as hydrogen is a more efficient fuel in comparison to the existing carbonaceous fossil fuels $[1,2]$. Despite many recent technological developments in the hydrogen-based fuel systems, it is still an enormous challenge to have a safe and efficient reversible hydrogen storage system at ambient conditions [2]. One possible way for hydrogen storage is an efficient and controllable adsorption/desorption system. Carbon based materials, such as carbon nanotubes and graphene, appear promising for such a purpose due to their large surfaces and light weight. It is known that there are two different hydrogen storage forms: in molecular form (through physical adsorption) or in atomic form (through chemical adsorption). Although several mechanisms of hydrogen storage through both physisorption and chemisorption have been proposed [3-13], most of these efforts are far to reach the target of $6 \mathrm{wt} \%$ and reversible hydrogen uptake and release for commercial

\begin{abstract}
sociatively adsorbed on $\mathrm{N}$-doped graphene spontaneously in the presence of a perpendicular electric field $F$. After adsorption, $\mathrm{H}$ atoms diffuse on $\mathrm{N}$-doped graphene surface with low barrier and the graphene can be fully hydrogenated. By removing the electric filed, the stored hydrogen can be released efficiently under ambient conditions. Therefore, $\mathrm{N}$-doped graphene is a promising hydrogen storage material with both easy hydrogen storage and release, and the storage capacity is up to $6.73 \mathrm{wt} \%$. The electric field can act as a switch for hydrogen uptake/release processes.
\end{abstract}

applications specified by U.S. Department of Energy (DOE).

In this work, only atomic hydrogen storage on graphene will be considered. Due to the high energy barrier for $\mathrm{H}_{2}$ molecules dissociative adsorption on graphene [10], based on density functional theory (DFT) calculations, different methods, such as applying external electric field and doping nitrogen into graphene, will be considered to lower the barrier. Hydrogen diffusion and hydrogen release from the hydrogen storage material will be also investigated by DFT calculations.

\section{Computational methodology}

The DFT calculations were performed using the DMOL3 code [14]. The generalized gradient approximation (GGA) with revised Perdew-Burke-Ernzerhof (RPBE) functional was employed as the exchange-correlation functional [15]. A double numerical plus polarization (DNP) was used as the basis set, while the DFT semicore pseudopotentials (DSPP) core treatment was employed to include 
relativistic effects that replaces core electrons by a single effective potential. Spin polarization was considered in the calculations. The convergence tolerance of the energy was set to $10^{-5} \mathrm{Ha}(1 \mathrm{Ha}=27.21 \mathrm{eV})$, and the maximum allowed force and displacement were $0.02 \mathrm{Ha}$ and $0.005 \AA$, respectively. To investigate the dissociative adsorption and diffusion pathways of the hydrogen on graphene surface and hydrogen release from graphene, linear synchronous transition/quadratic synchronous transit (LST/QST) [16] and nudged elastic band (NEB) [17] tools in the DMOL3 code were used. These methodologies have been demonstrated as fantastic tools to search for the structure of the transition state (TS) and the minimum energy pathway. In the simulations, three-dimensional periodic boundary conditions were imposed, and all the atoms are allowed to relax. We minimized the interlayer interaction by allowing a vacuum width of $18 \AA$ normal to the layer. The supercell used to investigate the hydrogen dissociative adsorption on graphene is shown in Fig. 1.

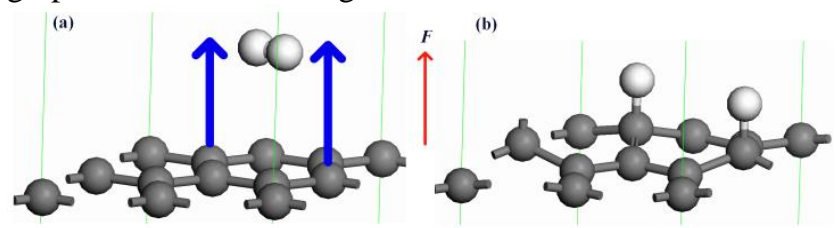

Figure 1 The initial (panel a) and final (panel b) structures for a $\mathrm{H}_{2}$ molecule dissociative adsorption on graphene. In this figure we show a $2 \times 2$ supercell where the gray and white balls are carbon and hydrogen atoms, respectively. The direction of the electric field $F$ is indicated by the arrow.

\section{Results and discussion \\ 3.1 Dissociative adsorption on pristine gra- phene}

For the case of molecular hydrogen adsorbed on graphene, Fig. 1(a) shows the favourite atomic structure with the distance between the $\mathrm{H}_{2}$ molecule and the graphene layer $d_{\mathrm{H} 2 \text {-graphene }}=2.612 \AA$, and adsorption energy $E_{\mathrm{b}-\mathrm{H} 2}=$ $-0.153 \mathrm{eV}$, which are consistent with other simulation results of $d_{\mathrm{H} 2 \text {-graphene }}=2.635 \AA$ and $E_{\mathrm{b}-\mathrm{H} 2}=-0.159 \mathrm{eV}$ in Ref. [8] and $d_{\mathrm{H} 2 \text {-graphene }}=2.8 \AA$ and $E_{\mathrm{b}-\mathrm{H} 2}=-0.133 \mathrm{eV}$ in Ref. [18]. For the case of atomic hydrogen adsorption on graphene, the favorable configuration is two $\mathrm{H}$ atoms adsorbed on two face-by-face carbon atoms in the same hexagon as shown in Fig. 1(b), which is consistent with the reported DFT result [19]. The $\mathrm{C}-\mathrm{H}$ bond length $l_{\mathrm{C}-\mathrm{H}}$ is 1.125 $\AA$ with binding energy $E_{\mathrm{b}-\mathrm{H}}=-2.184 \mathrm{eV}$, which agrees with another DFT result $l_{\mathrm{C}-\mathrm{H}}=1.13 \AA$ [19]. In addition, the $\mathrm{C}$ atoms bonded with the two $\mathrm{H}$ atoms move up toward the $\mathrm{H}$ atoms with about $0.32 \AA$ and the $\mathrm{C}-\mathrm{C}$ bond length $l_{\mathrm{C}-\mathrm{C}}$ is $1.493 \AA$, which is similar to the $s p^{3}$ bond length of $1.53 \AA$ in diamond and is much longer than $1.420 \AA$ for the $s p^{2}$ carbon length. The reconstruction of the graphene layer was also reported by others where the $\mathrm{C}$ atoms bonded with the $\mathrm{H}$ atoms move out of the graphene plane by 0.35 A [19].
For atomic hydrogen storage application, we are interested in the chemical reaction of $\mathrm{H}_{2}$ dissociative adsorption on graphene, especially the reaction energy barrier and reaction energy. Taking the structure of Fig. 1(a) as the initial structure (IS) and that of Fig. 1(b) as the final structure (FS) for this reaction, after LST/QST and NEB calculations, the detailed reaction pathway is shown in Fig. 2. The corresponding atomic structures for transition state (TS) and minimum energy state State1 are also shown in the figure. Following the reaction coordinate in Fig. 2, the reaction energy barrier for a $\mathrm{H}_{2}$ molecule dissociative adsorption $E_{\mathrm{bar}}$ is $2.734 \mathrm{eV}$ and the reaction energy $E_{\mathrm{R}}$ is $0.828 \mathrm{eV}$. The result of $E_{\mathrm{bar}}$ is a little smaller than $3.3 \mathrm{eV}$ found by others [19]. Note that the energy barrier is defined as the energy difference between the initial structure and the transition state. At TS, the $\mathrm{H}_{2}$ molecule is dissociated into two free $\mathrm{H}$ atoms without any binding with the $\mathrm{C}$ atoms. After TS, the two $\mathrm{H}$ atoms bind with the $\mathrm{C}$ atoms and move to the exact top sites of the $\mathrm{C}$ atoms shown as the final structure. Therefore, this reaction can be divided into two steps: $\mathrm{H}_{2}$ molecule is dissociated into two free $\mathrm{H}$ atoms, and then the two $\mathrm{H}$ atoms bind with the two $\mathrm{C}$ atoms. Step one needs an energy of $2.734 \mathrm{eV}$ to overcome the necessary potential barrier and the second step releases an energy of $1.9 \mathrm{eV}$. So totally the reaction needs about 0.8 $\mathrm{eV}$, the dissociation of $\mathrm{H}_{2}$ is the rate-limiting step because of larger energy required for this step. In addition, it is reported that surface reaction occur at ambient temperature when $E_{\mathrm{bar}}<0.75 \mathrm{eV}$ [20]. Therefore, the dissociative adsorption of $\mathrm{H}_{2}$ molecules on pristine graphene is very difficult due to the high reaction barrier.

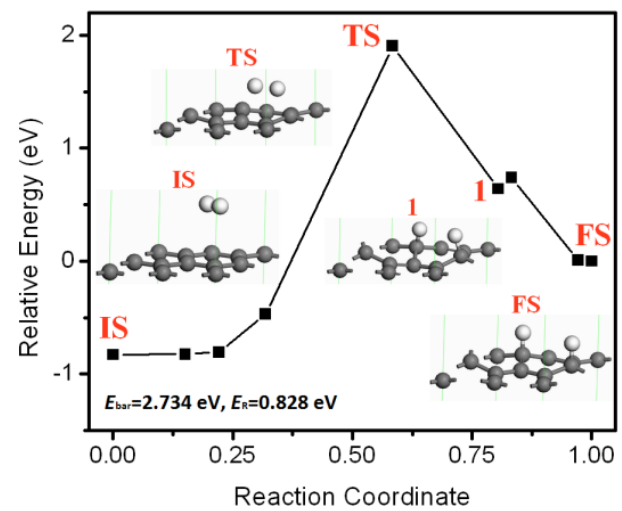

Figure 2 The reaction pathway of a $\mathrm{H}_{2}$ molecule that undergoes a dissociative adsorption on graphene. IS, TS, FS, and 1 represent initial structure, transition structure, final structure, energy minimum state State1, respectively. Their atomic structures are given by the inserts. The energy of FS is taken to be zero. $E_{\mathrm{bar}}$ is the energy barrier and $E_{\mathrm{R}}$ is the reaction energy.

\subsection{Dissociative adsorption on $\mathrm{N}$-doped gra-} phene

Using $a b$ initio calculations, doing $\mathrm{N}$ into carbon nanotubes (CNTs) was reported to reduce the energy barrier of hydrogen molecule dissociative adsorption [21]. In addi- 
tion, $\mathrm{N}$-doped graphene was prepared by carrying out arc discharge of graphite electrodes in the presence of $\mathrm{H}_{2}, \mathrm{He}$, and $\mathrm{NH}_{3}$ [22], chemical vapour deposition [23], and through electrothermal reaction with ammonia [24]. Therefore, $\mathrm{N}$ atoms are considered to be doped into graphene to investigate the effect on reducing the hydrogen dissociative adsorption energy barrier. One of the $\mathrm{C}$ atoms in Fig. 1 is replaced by a $\mathrm{N}$ atom. After geometry optimization, the Ndoped graphene still keeps planar with a slight deformation with $\mathrm{C}-\mathrm{N}$ bond length $l_{\mathrm{C}-\mathrm{N}}=1.41 \AA$ comparing to $l_{\mathrm{C}-\mathrm{C}}=$ $1.42 \AA$ in pristine graphene. The binding energy $E_{\mathrm{b}-\mathrm{N}}$ between $\mathrm{N}$ and the $\mathrm{C}$ atom in graphene can be also determined by the calculation, which is as strong as -12.65 $\mathrm{eV} /$ atom, while the $\mathrm{C}-\mathrm{C}$ binding energy was reported to be $-9.55 \mathrm{eV} /$ atom [25]. The structure after $\mathrm{N}$ doping is shown in the inserts of Fig. 3.
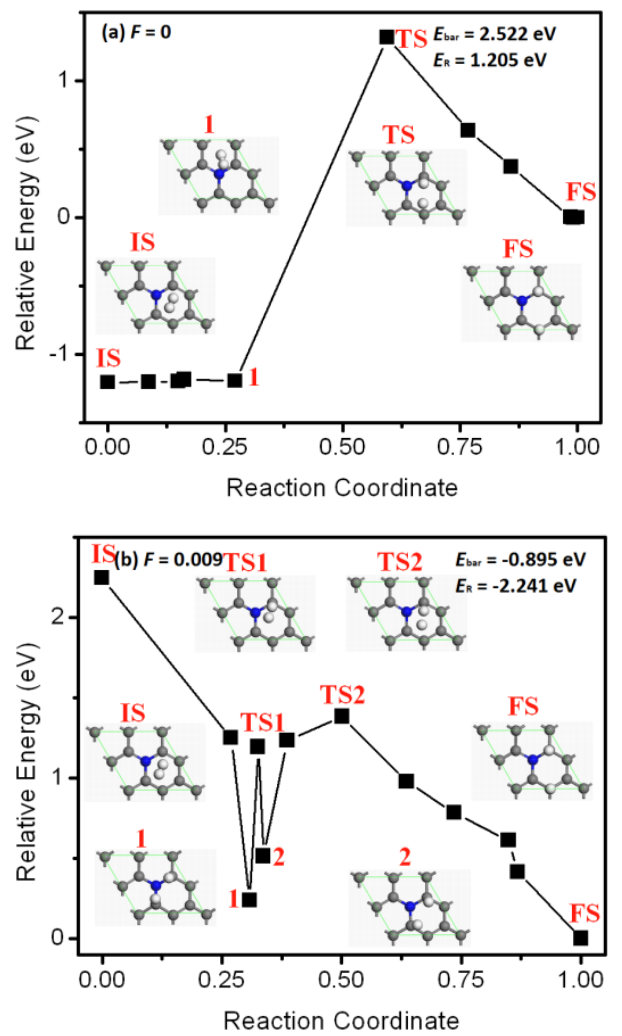

Figure 3 The reaction pathway of a $\mathrm{H}_{2}$ molecule that undergoes a dissociative adsorption on $\mathrm{N}$-doped graphene without electric field (a) and with an electric field of 0.009 au. Their atomic structures are given by the inserts. The blue balls are $\mathrm{N}$ atoms.

The dissociative adsorption pathway of a $\mathrm{H}_{2}$ molecule on the N-doped graphene is shown in Fig. 3(a). It is shown that the reaction barrier $E_{\mathrm{bar}}$ is $2.522 \mathrm{eV}$, which is a little smaller than that in pristine graphene $2.734 \mathrm{eV}$. This is also constant with the reported result that doping $\mathrm{N}$ into CNTs could reduce the hydrogen dissociative adsorption barrier [21]. However, the reaction energy barrier is still too high to go through at room temperature. Alternative method should be considered to further reduce the barrier.
It is known that an electric field $F$ can effectively modify the properties of low-dimensional system [26], including 1D quantum wires [27], and 2D graphene layers [28, 29]. For example, Zhou et al. found that the H-C bond length in graphane could increase with the intensity of an electric field, and finally the $\mathrm{H}$ atoms at one side of graphene would be desorbed if further increasing the electric field [29]. The mechanism is believed that a perpendicular electric field would lead to a polarization of the charge density, which is considered to be effective for the dissociative adsorption of hydrogen molecules on graphene here as well. To investigate the effect of an electric field on the hydrogen dissociative adsorption on $\mathrm{N}$-doped graphene, an electric field is applied to the N-doped graphene system. The direction of the electric field is normal to the surface of graphene, which is shown as the arrow direction in Fig. 1. It is found that a positive electric field reduces the barrier significantly. If we further increase $F$ above 0.009 au ( $1 \mathrm{au}$ $\left.=5.14 \times 10^{11} \mathrm{~V} / \mathrm{m}\right)$, no barrier is found, which means that the hydrogenation reaction occurs automatically. Note that the reaction barrier becomes negative when $F>0.005$ au. We also apply a negative electric field in this system, however, the barrier increases under the negative electric field. It is understandable that reversing the electric field has an opposite effect on the energy barrier for hydrogen dissociative adsorption due to the polarization effect of the electric field.

The reaction pathway in the presence of an electric field $F=0.009$ au is shown in Fig. 3(b), where the energies of the initial structure, transition structures, final structures, reaction barriers and reaction energies are also provided. It is interesting to notice that the reaction barrier for a $\mathrm{H}_{2}$ molecule dissociative adsorbed on $\mathrm{N}$-doped graphene is negative, i.e., $-0.895 \mathrm{eV}$. According to the pathway, the initial structure is reconstructed under the electric field from IS to State 1, where the $\mathrm{H}_{2}$ molecule is dissociated into $2 \mathrm{H}$ atom and bind with two $\mathrm{C}$ atoms besides the doped $\mathrm{N}$ atom. However, this configuration is not the energy minimum structure. To achieve the energy minimum structure, which is shown as FS in Fig. 3(d), there are two barriers, i.e., from State1 to TS1 with a barrier of $0.966 \mathrm{eV}$ and from State2 to TS2 with a barrier of $0.801 \mathrm{eV}$, as shown in Fig. 3(b). It is known from Fig. 3(b) the energy from the exothermic process of IS to State1 is sufficient to support the endothermic process from State1 to TS2. Therefore, $\mathrm{H}_{2}$ molecules can be dissociatively adsorbed on N-doped graphene automatically under the electric field. The corresponding atomic structures of IS, State1, State2, TS1, TS2 and FS are also provided in the inserts of Fig. 3. After State1, one of the two $\mathrm{H}$ atoms desorbs from the graphene surface, adjusts its position and move to the $\mathrm{C}$ atom, which is face to the $\mathrm{C}$ atom that the other $\mathrm{C}$ atom is binding with. After the two transition states, the $\mathrm{H}$ atom binds with the $\mathrm{C}$ atom and the minimum structure FS is achieved. Note that the two $\mathrm{H}$ atoms are adsorbed at two $\mathrm{C}$ atoms that are not symmetric as shown as FS in Fig. 3(b). In other words, one 
$\mathrm{H}$ atom is adsorbed onto the $\mathrm{C}$ atom that is the nearest to the $\mathrm{N}$ atom, the other one is adsorbed on the $\mathrm{C}$ atom that is the second nearest to the $\mathrm{N}$ atom, not another $\mathrm{C}$ atom that the nearest to the $\mathrm{N}$ atom. This is because the adsorption of the two $\mathrm{H}$ atoms does not occur at the same time as shown in the reaction pathway of Fig. 3(b). The adsorption of the first $\mathrm{H}$ atom changes the electric distribution, thus chemical potential and reactivity of each $\mathrm{C}$ atom. Therefore, the electric field can act as a catalyst to reduce the hydrogenation barrier of $\mathrm{N}$-doped graphene, and $\mathrm{H}_{2}$ molecules dissociative adsorption on $\mathrm{N}$-doped graphene occurs spontaneously in the presence of the electric field.

3.3 Hydrogen diffusion and release on/from $\mathrm{N}$ doped graphene

It is known that $\mathrm{H}_{2}$ molecules can be spontaneously dissociatively adsorbed on $\mathrm{N}$-doped graphene under a perpendicular applied electric field. These dissociated $\mathrm{H}$ atoms prefer to be chemically adsorbed on the $\mathrm{C}$ atoms near the doped $\mathrm{N}$ atom. To be an efficient hydrogen storage material, $\mathrm{N}$-doped graphene is required to capture as much hydrogen as possible. Therefore, it is desirable that the adsorbed $\mathrm{H}$ atoms bonded with the $\mathrm{C}$ atoms near the doped $\mathrm{N}$ atom can diffuse to the other $\mathrm{C}$ atomic sites in order to achieve fully hydrogenated graphene. Subsequently, the adsorbed $\mathrm{H}$ atoms are required to desorb from the surface and form $\mathrm{H}_{2}$ molecules easily to realise hydrogen release as fuel. Therefore, we are interested in the diffusion behaviours of hydrogen on N-doped graphene, hydrogen desorption and $\mathrm{H}_{2}$ formation will also be studied to understand the release procedure.

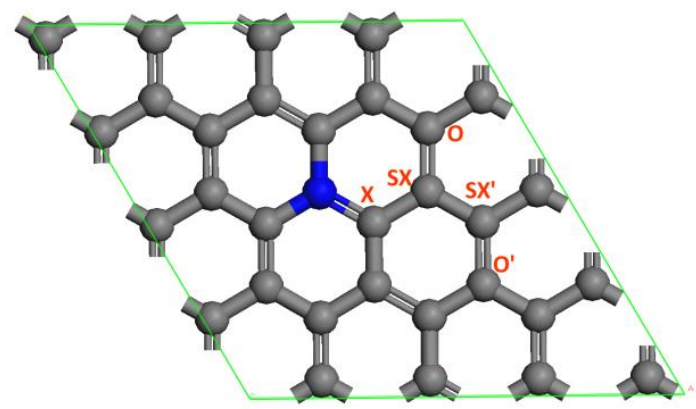

Figure 4 Supercell of N-doped graphene used for the diffusion and desorption calculations. The letters refer to the sites of an adsorbed $\mathrm{H}$ atom.

To simplify the investigation, we first consider the diffusion of a single $\mathrm{H}$ atom on $\mathrm{N}$-doped graphene. There are 5 different possible adsorption sites as indicated by the letters in Fig. 4. After geometry relaxation, it is found that the $\mathrm{H}$ atom adsorbed on the $\mathrm{C}$ atom at site $\mathrm{X}$ has the lowest energy, i.e. this is the most favourable configuration for a single $\mathrm{H}$ atom adsorption and it is considered as the base structure for studying the diffusion of an individual $\mathrm{H}$ atom on N-doped graphene. As shown in Fig. 4, there are four possible diffusion pathways: $\mathrm{X} \rightarrow \mathrm{SX}, \mathrm{X} \rightarrow \mathrm{O}, \mathrm{X} \rightarrow \mathrm{SX}^{\prime}$ and $\mathrm{X} \rightarrow \mathrm{O}^{\prime}$. After LST/QST and NEB calculations, the corresponding diffusion barrier and reaction energy are given in Table 1. It is found that the energy barrier for a single $\mathrm{H}$ atom diffusion on the $\mathrm{N}$-doped graphene surface is quite high, around $2.5 \mathrm{eV}$. In addition, the diffusion would increase the energy of the system. Therefore, $\mathrm{H}$ atoms are quite stable on the $\mathrm{N}$-doped graphene surface comparing with the case of a single $\mathrm{H}$ atom adsorbed on pristine graphene, which has a diffusion barrier of $\sim 1 \mathrm{eV}$ [30].

Table 1 Diffusion barriers and diffusion energy of four possible diffusion pathways for a single $\mathrm{H}$ atom on $\mathrm{N}$-doped graphene with and without an electric field. $E_{\mathrm{R}}$ and $E_{\mathrm{bar}}$ are diffusion energy and diffusion barrier, respectively.

\begin{tabular}{cccc}
\hline Electric field (a.u.) & Pathway & $E_{\mathrm{R}}(\mathrm{eV})$ & $E_{\mathrm{Bar}}(\mathrm{eV})$ \\
\hline 0 & $\mathrm{X} \rightarrow \mathrm{SX}$ & 0.61 & 2.21 \\
& $\mathrm{X} \rightarrow \mathrm{O}$ & 0.27 & 2.48 \\
& $\mathrm{X} \rightarrow \mathrm{SX^{ \prime }}$ & 0.58 & 2.48 \\
$\mathrm{X} \rightarrow \mathrm{O}^{\prime}$ & 0.66 & 2.61 \\
\hline 0.01 & $\mathrm{X} \rightarrow \mathrm{SX}$ & 0.55 & 0.47 \\
& $\mathrm{X} \rightarrow \mathrm{O}$ & 0.29 & -0.094 \\
& $\mathrm{X} \rightarrow \mathrm{S} \mathrm{X}^{\prime}$ & 0.56 & -0.10 \\
& $\mathrm{X} \rightarrow \mathrm{O}^{\prime}$ & 0.62 & 0.62 \\
\hline
\end{tabular}

However, the high stability of $\mathrm{H}$ atoms on graphene surface is not favourable for hydrogen storage due to the reversible hydrogen uptake and release requirement. It is known that $\mathrm{H}_{2}$ molecules can dissociatively adsorbed on $\mathrm{N}$-doped graphene in the presence of an electric field with $F>0.009$ au. Therefore, the diffusion behaviour under $F$ is interesting for hydrogen storage application. Table 1 also lists the results of the diffusion along four different pathways under $F=0.01$ au. It is shown that the diffusion barrier $E_{\text {bar }}$ decreases significantly for all four pathways, and is even negative for the diffusions of $\mathrm{X} \rightarrow \mathrm{O}$ and $\mathrm{X} \rightarrow \mathrm{SX}^{\prime}$, while diffusion energy $E_{\mathrm{R}}$ decreases slightly as well. This indicates that a single $\mathrm{H}$ atom diffusion on the $\mathrm{N}$-doped graphene is favourable under the electric field.

Recently, the diffusion barriers of transition-metal (TM) atoms on graphene were reported to be in the range of 0.2 $0.8 \mathrm{eV}$. However, if the TM adatoms are coupled to a vacancy, the diffusion barrier would increase to $2.1-3.1 \mathrm{eV}$ [31]. A similar barrier enhancement was also predicted in the case of $\mathrm{H}$ atom diffusion at the graphene/graphane interface [32]. Therefore, specific defects, such as doping, vacancies and adatoms, will significantly affect the diffusion behaviour of $\mathrm{H}$ on graphene. In this case, the diffusion of two $\mathrm{H}$ atoms on $\mathrm{N}$-doped graphene will be studied to determine the effect of another $\mathrm{H}$ atom on hydrogen diffusion. After LST/QST and NEB calculations, it is found that the diffusion barrier is less than $0.31 \mathrm{eV}$ under the electric field of $0.01 \mathrm{au}$, which can be easily overcome at room temperature. In addition, it was reported that the ease of adsorbate diffusion on graphene strongly depends on the carrier density of graphene [33]. Through a Mulliken analysis in this study, it is known that the $\mathrm{H}$ atoms transfer 
electrons to the $\mathrm{N}$-doped graphene layer. Therefore, with the presence of more $\mathrm{H}$ atoms, the carrier density of graphene is higher, and the $\mathrm{H}$ diffusion is much easier. Due to the low barrier of $\mathrm{H}$ atom diffusion on $\mathrm{N}$-doped graphene under the electric field, $\mathrm{N}$-doped graphene is believed to be fully hydrogenated under the electric field, and all the $\mathrm{H}$ atoms are adsorbed at a single side of graphene, i.e. this is the uptake process for hydrogen storage.

Then we are interested in the study of how do the $\mathrm{H}$ atoms release from the $\mathrm{N}$-doped graphene and combine into $\mathrm{H}_{2}$ molecules. This desorption reaction can be also investigated by LST/QST and NEB calculations. First, we study the case of $2 \mathrm{H}$ atoms desorption from the $\mathrm{N}$-doped graphene and combine into $1 \mathrm{H}_{2}$ molecule after removing the electric field. After the structure relaxation, the favourite configurations before and after desorption are shown as IS and FS in Fig. 5, respectively. Before desorption, the two $\mathrm{H}$ atoms bind with two nearest $\mathrm{C}$ atoms. This is different from the structure obtained in Ref. [11] where one $\mathrm{H}$ atom binds with the $\mathrm{C}$ nearest to the $\mathrm{N}$, the other one binds with the $\mathrm{C}$ the second nearest to the $\mathrm{N}$, due to the different simulation supercell size in the two studies, which leads to different interaction between neighbour supercells. After desorption, the $\mathrm{H}_{2}$ molecule takes the hollow site of the $\mathrm{C}$ ring with the doped $\mathrm{N}$ atom. This is consistent to the reported result [11]. Taking the configuration before desorption as the initial structure and that after desorption as the final structure for the reaction, the detail reaction pathway is shown in Fig. 5. However, the desorption barrier $E_{\mathrm{bar}}=$ $2.12 \mathrm{eV}$ is quite high; release of hydrogen in this case is difficult to happen.
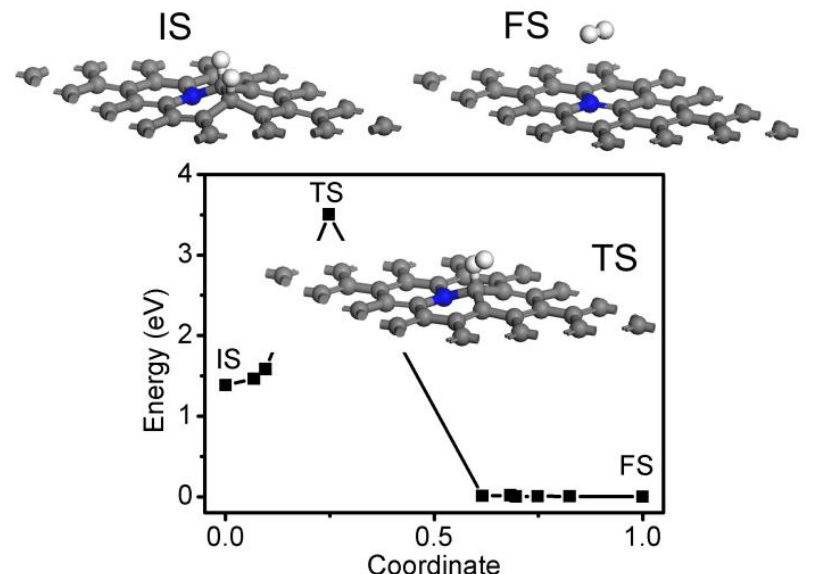

Figure 5 Detailed reaction pathway for $2 \mathrm{H}$ atoms desorption from $\mathrm{N}$-doped graphene and combination into a $\mathrm{H}_{2}$ molecule, where the structure of IS, TS and FS are also given.

As discussed above, the presence of specific defects, such as doping, vacancies and adatoms, will significantly affect the chemical potential of $\mathrm{C}$ atom in graphene. In addition, the hydrogen is expected to be released from the fully hydrogenated N-doped graphene. Therefore, we are also interested in the situation where a third $\mathrm{H}$ atom is pre- sent near the two $\mathrm{H}$ atoms. The favourite configurations before and after desorption are shown in Fig. 6 as IS and FS, respectively. The detailed desorption pathway is also shown in this figure. As shown in Fig. 6, the $2 \mathrm{H}$ atoms prefer to desorb from the $\mathrm{N}$-doped graphene and combine into a $\mathrm{H}_{2}$ molecule with the presence of a third $\mathrm{H}$ atom as shown by State1. This means that the $\mathrm{C}-\mathrm{H}$ bond in this case is unstable and hydrogen can released in the form of the $\mathrm{H}_{2}$ molecule spontaneously when the hydrogen concentration is higher than $0.5 \mathrm{wt} \%$ where only $2 \mathrm{H}$ atoms left in the supercell. Subsequently, this free $\mathrm{H}_{2}$ molecule can diffuse on the $\mathrm{N}$-doped graphene surface with a very low energy barrier (insert of Fig. 6), where $E_{\text {bar }}^{\prime}$ and $E_{\mathrm{R}}^{\prime}$ are the actual reaction barrier and reaction energy, which are defined as the energy differences between TS and State1, State 1 and FS, respectively. When the hydrogen concentration is higher, hydrogen release is expected to be easier due to the weaker $\mathrm{C}-\mathrm{H}$ bonds and diffusion barrier as discussed above.

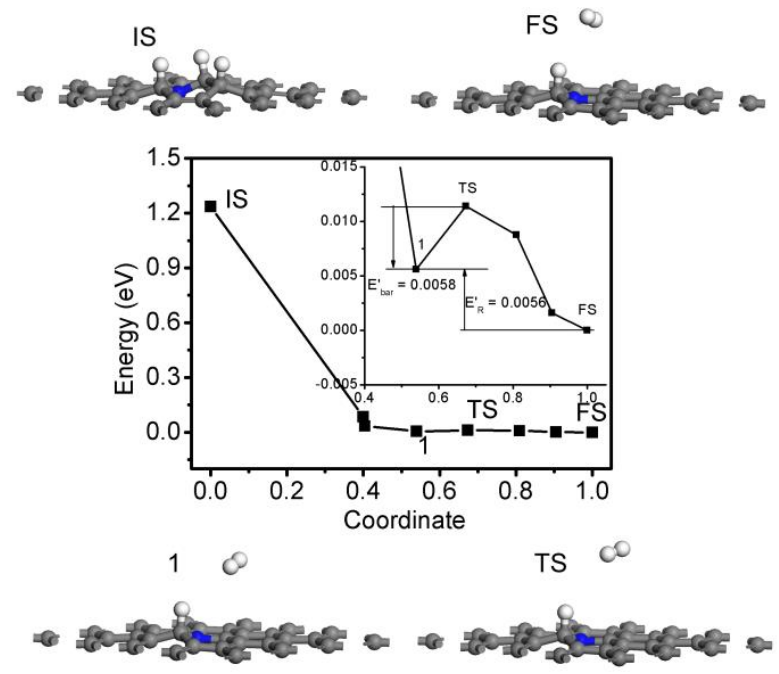

Figure 6 Detailed reaction pathway for the desorption of $2 \mathrm{H}$ atoms from $\mathrm{N}$-doped graphene and combination into a $\mathrm{H}_{2}$ molecule with presence of a third $\mathrm{H}$ atom nearby.

Therefore, the highest possible hydrogen storage capacity of $\mathrm{N}$-doped graphene in the absence of an electric field is $\sim 0.5 \mathrm{wt} \%$. In the presence of the applied electric field, due to the low dissociated adsorption barrier for $\mathrm{H}_{2}$ molecules on $\mathrm{N}$-doped graphene and low diffusion barrier of $\mathrm{H}$ atoms on the $\mathrm{N}$-doped graphene surface, the $\mathrm{N}$-doped graphene is expected to be fully hydrogenated and the hydrogen weight ratio can reach up to $7.23 \mathrm{wt} \%$, which is higher than the target of $6 \mathrm{wt} \%$ set by DOE. After removing the electric field, hydrogen atoms in fully hydrogenated $\mathrm{N}$-doped graphene release easily until the hydrogen weight ratio of $0.5 \mathrm{wt} \%$. In this way, the hydrogen storage capacity of $\mathrm{N}$-doped graphene is $6.73 \mathrm{wt} \%$, while the electric field is the switch for hydrogen uptake/release processes. In addition, the $0.5 \mathrm{wt} \%$ hydrogen left on $\mathrm{N}$-doped graphene can be further removed by increasing temperature. 


\section{Conclusions}

Doping $\mathrm{N}$ into graphene and applying an electric field can reduce the energy barrier of hydrogen molecules dissociative adsorption on graphene, thus facilitating the hydrogenation process of graphene. In the presence of the same electric field, the dissociatively adsorbed $\mathrm{H}$ atoms can diffuse on the N-doped graphene easily with low barrier. Therefore, a fully hydrogenated N-doped graphene can be obtained with atomic hydrogen storage capacity of 7.23 wt $\%$. By removing the electric field, the stored hydrogen can be released efficiently without any barrier when the hydrogen concentration is higher than $0.5 \mathrm{wt} \%$. Therefore, $\mathrm{N}$-doped graphene is a promising atomic hydrogen storage material with a storage capacity of up to $6.73 \mathrm{wt} \%$, and with reversible hydrogen storage/release manipulated by the electric field.

Acknowledgements Financial supports of the ViceChancellor's Postdoctoral Research Fellowship Program (SIR50/PS19184) and the Goldstar Award (RG124422) from the University of New South Wales are acknowledged.

\section{References}

[1] R. Coontz and B. Hanson, Science 305, 957 (2004).

[2] L. Schlapbach and A. Züttel, Nature 414, 353 (2001).

[3] K. Chandrakumar and S. Ghosh, Nano Lett. 8, 13 (2008).

[4] W. Deng, X. Xu, and W. Goddard, Phys. Rev. Lett. 92, 166103 (2004).

[5] E. Klontzas, A. Mavrandonakis, E. Tylianakis, and G. Froudakis, Nano Lett. 8, 1572 (2008).

[6] G. Mpourmpakis, E. Tylianakis, and G. Froudakis, Nano Lett. 7, 1893 (2007).

[7] A. Nikitin, X. Li, Z. Zhang, H. Ogasawara, H. Dai and A. Nilsson, Nano Lett. 8, 162 (2008).

[8] Z. M. Ao, Q. Jiang, R. Q. Zhang, T. T. Tan, and S. Li, J. Appl. Phys. 105, 074307 (2009).

[9] Z. M. Ao and F. M. Peeters, Phys. Rev. B 91, 205406 (2010).

[10]Z. M. Ao and F. M. Peeters, Appl. Phys. Lett. 96, 253106 (2010).

[11]Z. M. Ao and F. M. Peeters,J. Phys. Chem. C 114, 14503 (2010).

[12] Z. M. Ao, A. D. Hernandez-Nieves, F. M. Peeters, and S. Li, Phys. Chem. Chem. Phys. 14, 1463 (2012).

[13] A. Du, Z. Zhu, and S. C. Smith, J. Am. Chem. Soc. 132, $2876(2010)$.

[14]B. Delley, J. Chem. Phys. 113, 7756 (2000).

[15]B. Hammer, L. B. Hanse, and J. K. Nфrskov, Phys. Rev. B 59, 7413 (1999).

[16]T. A. Halgren and W. N. Lipscomb, Chem. Phys. Lett. 49, 225 (1977)

[17]G. Henkelman and H. Jonsson, J. Chem. Phys. 113, 9978 (2000).

[18]Y. Okamoto and Y. Miyamoto, J. Phys. Chem. B 105, 3470 (2001).

[19]Y. Miura, H. Kasai, W. Dino, H. Nakanishi, and T. Sugimoto, J. Appl. Phys. 93, 3395 (2003)

[20]C. Shang and Z. P. Liu, J. Am. Chem. Soc. 133, 9938 (2011).
[21]Z. Zhang and K. Cho, Phys. Rev. B 75, 075420 (2007)

[22]L. S. Panchakarla, K. S. Subrahmanyam, S. K. Saha, A. Govindaraj, H. R. Krishnamurthy, U. V. Waghmare, and C. N. R. Rao, Adv. Mater. 21, 4726 (2009).

[23]D. Wei, Y. Liu, Y. Wang, H. Zhang, L. Huang, and G. Yu, Nano Lett. 9, 1752 (2009).

[24]X. Wang, X. Li, L. Zhang, Y. Yoon, P. K. Weber, H. Wang, J. Guo, and H. Dai, Science 324, 768 (2009).

[25]J. O. Sofo, A. S. Chaudhari, and G. D. Barber, Phys. Rev. B 75, 153401 (2007).

[26]W. Liu and Q. Jiang, J. Comput. Theor. Nanos, 7, 2225 (2010).

[27]C. He, P. Zhang, Y. F. Zhu, and Q. Jiang, J. Phys. Chem. C 112, 9045 (2008).

[28]Z. M. Ao, W. T. Zheng, and Q. Jiang, Nanotechnology 19 , 275710 (2008)

[29]J. Zhou, M. M. Wu, X. Zhou, and Q. Sun, Appl. Phys. Lett. 95, 103108 (2009).

[30]L. F. Huang, M. Y. Ni, X. H. Zheng, W. H. Zhou, Y. G. Li, and Z. Zeng, J. Phys. Chem. C 114, 22636 (2010).

[31]A. V. Krasheninnikov, P. O. Lehtinen, A. S. Foster, P. Pyykko, and R. M. Nieminen, Phys. Rev. Lett. 102, 126807 (2009).

[32]Z. M. Ao, A. D. Hernandez-Nieves, F. M. Peeters, and S. Li, Appl. Phys. Lett. 97, 233109 (2010).

[33]A. M. Suarez, L. R. Radovic, E. Bar-Ziv, and J. O. Sofo, Phys. Rev. Lett. 106, 146802 (2011). 